\title{
Biological effects of non-ionizing electromagnetic fields to 27 GHz on sperm quality of Mytilus galloprovincialis
}

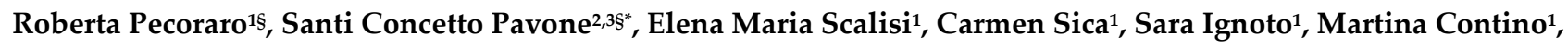 \\ Antonio Salvaggio ${ }^{4}$, Gino Sorbello ${ }^{2,3}$, Loreto Di Donato ${ }^{2,3 \S \$}$ and Maria Violetta Brundo $1 \$ \$^{*}$ \\ 1 Department of Biological, Geological and Environmental Science, University of Catania, Via Androne 81, \\ 95124, Catania, Italy, roberta.pecoraro@unict.it, elena.scalisi@phd.unict.it, carmensica93@gmail.com, \\ sara.ignoto@phd.unict.it, martina.contino@phd.unict.it, mariavioletta.brundo@unict.it \\ 2 Department of Electrical, Electronics, and Computer Engineering (DIEEI), University of Catania, Viale \\ Andrea Doria 6, 95125 Catania, Italy, santi.pavone@unict.it, gino.sorbello@unict.it, loreto.didonato@unict.it \\ 3 Consorzio Nazionale Interuniversitario per le Telecomunicazioni (CNIT), Viale G.P. Usberti, 181/A Pal. 3, \\ 43124 Parma, Italy, santi.pavone@unict.it, gino.sorbello@unict.it, loreto.didonato@unict.it \\ 4 Experimental Zooprophylactic Institute of Sicily “A. Mirri”, Via Gino Marinuzzi, 3, Palermo 90129, Italy, \\ antonio.salvaggio@izssicilia.it \\ $\S$ These authors have contributed equally to this work and share first authorship \\ ss. These authors have contributed equally to this work and share last authorship \\ * Correspondence: mariavioletta.brundo@unict.it; roberta.pecoraro@unict.it Tel.: +390957306039
}

\begin{abstract}
Recently, a rising use of wireless internet technologies has been demonstrated. The devices which use these technologies emit a considerable amount of electromagnetic radiation (EMFs) which could interact with the male reproductive system. The aim of this study was to investigate in vitro influence of electromagnetic fields a $27 \mathrm{GHz}$ on sperm quality in Mytilus galloprovincialis. The experiments were conducted with a no commercial high gain pyramidal horn antenna. Sperm samples, taken from sexually mature males of M. galloprovincialis, were placed in seawater. Once evaluated the number and quality of spermatozoa, sperm cells were exposed to electromagnetic fields. The effect of exposure was evaluated after 10, 20, 30 and 40 minutes with light microscope and using Eosin test. All the samples were performed in triplicate and statistical analysis was carried out by one-way ANOVA test. A significative decrease (30\%) in sperm motility was observed after 10 minutes of exposure and after 30 minutes all sperms were immobile and not vital. The measurement of the Oxidation Reduction Potential (sORP), which evaluates the oxidative damage on spermatozoa, shows how the exposure to $27 \mathrm{GHz}$ has increased the oxidant amount compared to the control groups. This study provides useful data on potential ecological impact of the EMFs on aquatic animals, that currently are poor investigated.
\end{abstract}

Keywords: Mussel; spermatozoa; millimeter waves; ROS

\section{Introduction}

With the roll-out of 5G mobile networks, significantly higher mobile broadband speeds and increasingly wider use of mobile data will be ensured. This is made possible by the use of additional higher frequency bands [1]. 5G aims to be the intersection of communications, from virtual reality to autonomous vehicles to the industrial internet and smart cities. Furthermore, 5G is considered to be the core technology for the Internet of Things (IoT), where machines communicate with machines [1]. At the same time, a change in exposure to electromagnetic fields (EMF) of humans and the environment is expected. 5G networks will work in different frequency bands and are divided into two different groups [2]. The first group, called Frequency Range 1 (FR1), includes the frequency bands below $6 \mathrm{GHz}$, some of which have already been used by previous standards but extended to cover new portions of the spectrum between $410 \mathrm{MHz}$ and 7125 
MHz. The second group, called Frequency Range 2 (FR2),includes the frequency range between $24.25 \mathrm{GHz}$ and $52.6 \mathrm{GHz}$ (millimeter waves or mmWave) and has a lower range but allows a wider available bandwidth than the bands of the FR1 group. The introduction of this new technology that operates in different frequency bands has attracted a significant amount of toxicity studies [3-5]. To date, however, only a few studies have been conducted on the frequencies that will be used by 5G. Tissue heating is the main mechanism of interaction between radiofrequency fields and the human body. The levels of radiofrequency exposure of current technologies cause a negligible increase in temperature in the human body. As the frequency increases, there is less penetration into the body's tissues and the absorption of energy becomes more limited to the surface of the body (skin and eyes). Provided that the overall exposure remains below international guidelines, there are no consequences for public health [6]. WHO is conducting a health risk assessment from radio frequency exposure, covering the full range of radio frequencies, including 5G, reviewing literature data on potential health risks from exposure to 5G. Unfortunately, little is known about the effect this new technology could have on coastal marine species. Owing to increasing pressure on the environment by humans, biodiversity loss has become one of the greatest environmental concerns. Habitat destruction and overexploitation represent the greatest stressors to marine biodiversity, but excessive anthropization, including the installation of antennas or of repeaters, can also be a threat especially for reproduction of many species [7]. The aim of this study was to investigate in vitro influence of electromagnetic fields to $27 \mathrm{GHz}$ on sperm quality of bivalve mollusk Mytilus galloprovincialis. The experiments were conducted with a no commercial high gain pyramidal horn antenna. The effects of exposure were measured evaluating sperms motility and viability. We also estimated oxidative stress with MiOXSYS System for evaluating the oxidative damage of the spermatozoa due to free radicals.

\section{Materials and Methods}

\subsection{Exposure Setup Description and Numerical Dosimetry}

The experiments at $27 \mathrm{GHz}$ were conducted by using an in house produced high gain pyramidal horn antenna feeder for parabolic antennas and satellite power pattern measurements applications. The dimension of the antenna aperture are $8.02 \times 6.02 \mathrm{~cm}$ and the maximum gain is $24.9 \mathrm{dBi}$. Moreover, the antenna is fed by a RF signal generation (R\&S SMB100A) with $+23 \mathrm{dBm}$ output power through a coaxial cable that introduce attenuation of $3 \mathrm{~dB}$. The distance between the antenna aperture and Petri dishes was set to $15 \mathrm{~cm}$ assuming normal incidence of the antenna main beam, in order to ensure density incident power level comparable with the exposure limit imposed by the international guidelines [6]. To evaluate the specific absorption rate (SAR), a numerical simulation has been performed by means of the electromagnetic simulator CST Microwave Studio.

\subsection{Experimental Procedure}

Mytilus galloprovincialis were purchased from a mussel farm in Sicily, placed in plastic bags with sea water and immediately transported to the Catania University's Laboratories. We proceeded then to remove from the sample the individuals with obvious signs of breakage of the shell. Once verified their maturity, the mussels were cleaned from debris and epibionts by manual scraping of the shell, rinsed quickly with water and stabulated for 1 hour before experiments. Mussels selected (20 individuals) were induced to spawn eggs and sperm by applying a protocol of thermal stimulation (heat shock). Mussels were placed at temperature of $4^{\circ} \mathrm{C}$ for 3-4 hours and then they were transferred into a tank containing water heated at $28^{\circ} \mathrm{C}$. The water used for the stimulation had a salinity of $30+1 \%$ and $\mathrm{pH} 8.3$ and it had been filtered with a $20 \mu \mathrm{m}$ filter. After thirty minutes most of the specimens had opened the valve and resumed filtration and they 
were transferred to a second tank containing sea water at a temperature of $18^{\circ} \mathrm{C}$. Once initiated the gametes spawning, the specimens were immediately removed and placed in an aquarium. Spermatozoa were placed in 6-well microplates in $5 \mathrm{ml}$ seawater/well. Control samples (negative control) were incubated only with seawater. A controlled room temperature has allowed to maintain $26 \pm 1^{\circ} \mathrm{C}$ in wells. Ten replications were performed.

\subsection{Motility analysis}

We measured the motility dividing spermatozoa into two categories: motility and immotility. The analysis was made by placing $10 \mu \mathrm{l}$ of sperms sample on a slide and observing under an optical microscope at x400 magnification. We counted 100 spermatozoa at least.

\subsection{Vitality analysis}

The procedure involves the positioning of $10 \mu \mathrm{l}$ of sperms sample on a slide in which we added $10 \mu \mathrm{l}$ of Eosin $\mathrm{Y}(0.5 \%)$. The slides were observed under an optical microscope Leica DMLB at x400 magnification. At least 100 spermatozoa were counted. Dead spermatozoa were dyed in pink due to the loss of membrane integrity, compared to live spermatozoa that maintain their original coloring.

\subsection{Oxidative stress analysis with MiOXSYS System}

MiOXSYS System is based on the measurement of the Oxidation Reduction Potential (sORP), an overall index of the electron passage to which a biological component is subjected. The test is carried out by placing a small aliquot $(30 \mu \mathrm{l})$ of seminal fluid on a sensor previously inserted in the analyzer that applies a low voltage current. The electron activity is measured in millivolts $(\mathrm{mV})$.

\subsection{Statistical analyses}

Statistical analysis was carried out using one-way analysis of variance (ANOVA) with Tukey test for multiple comparations of means to determine difference of sperms motility and vitality between the unexposed and exposed samples.

\section{Results}

\subsection{Numerical dosimetry analyses}

SAR level and distribution are non-homogeneous among the sample and among the different Petri dishes, but as spermatozoa move randomly inside the sample and they are exposed to medium SAR values of $0.115 \mathrm{~W} / \mathrm{Kg}$ for the peripheral samples and about 0.18 $\mathrm{W} / \mathrm{Kg}$ for the innermost sample. However, the average level of SAR in the different numerical layer along $\mathrm{z}$ direction (sample depth) keeps very uniform. This is mainly due to the presence of the metallic screen under the Petri dishes. At the top layers of the samples, instead, different levels of SAR are expected (with respect to the innermost layers) due to strong electromagnetic discontinuities. However, these density power levels are below the recently released ICNIRP guidelines [6]. The dosimetric analysis suggests that no relevant thermal energy is deposited on the system by the MMW radiation, in fact temperature measurements of the aqueous sample during the water refilling, have sensed a temperature increase of $0.2^{\circ} \mathrm{C}$, with respect to the control samples. 


\subsection{Sperms vitality and motility analyses}

A significative decrement of sperm's vitality (Fig.1) was observed already after 10 minutes of exposure to $27 \mathrm{GHz}(<40 \%)$, compared to control samples. Eosin test showed a mortality of $90 \%$ of spermatozoa in all exposed samples already after 30 minutes (Fig. 2). We observed that electromagnetic fields irradiation induced also a significant decrease in sperm motility (Fig. 3) after 10 minutes of exposure (<40\%).

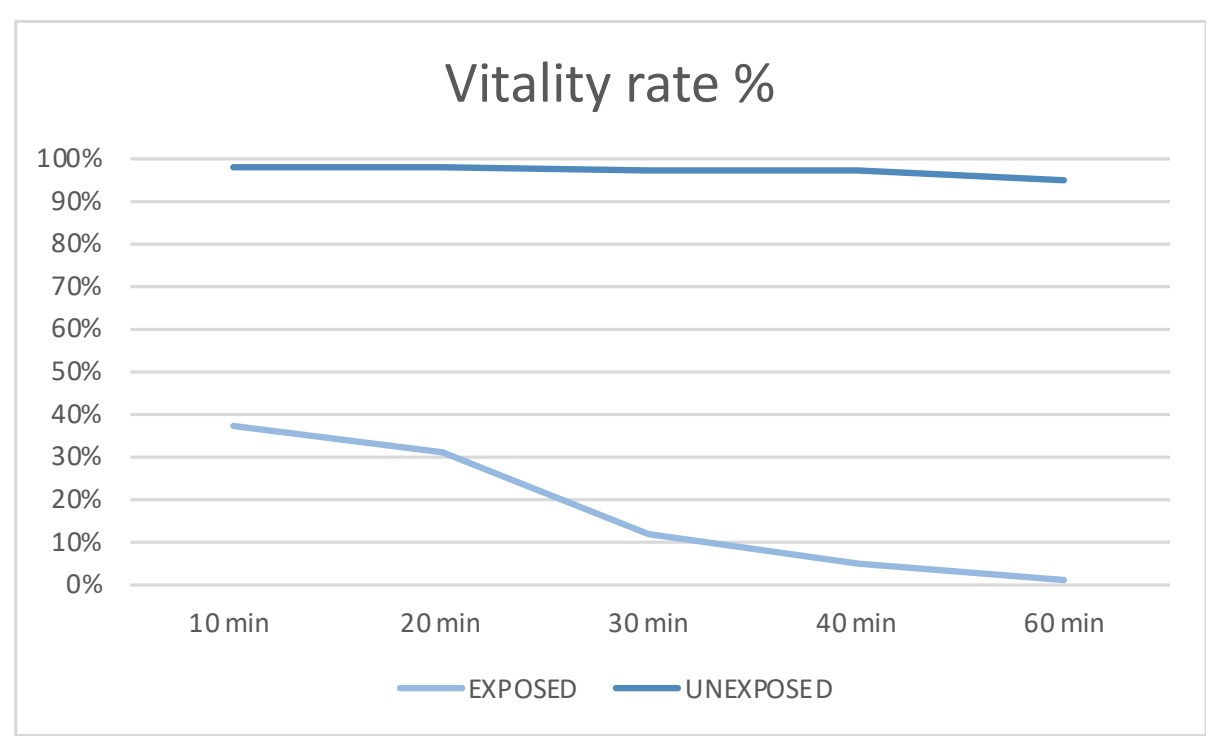

Figure 1. Temporal trends of the time-exposure decrease of sperms vitality to $27 \mathrm{GHz}$ frequency. The data represent the mean of observation performed in tenfold by same observer to avoid subjective differences in vitality evaluation.

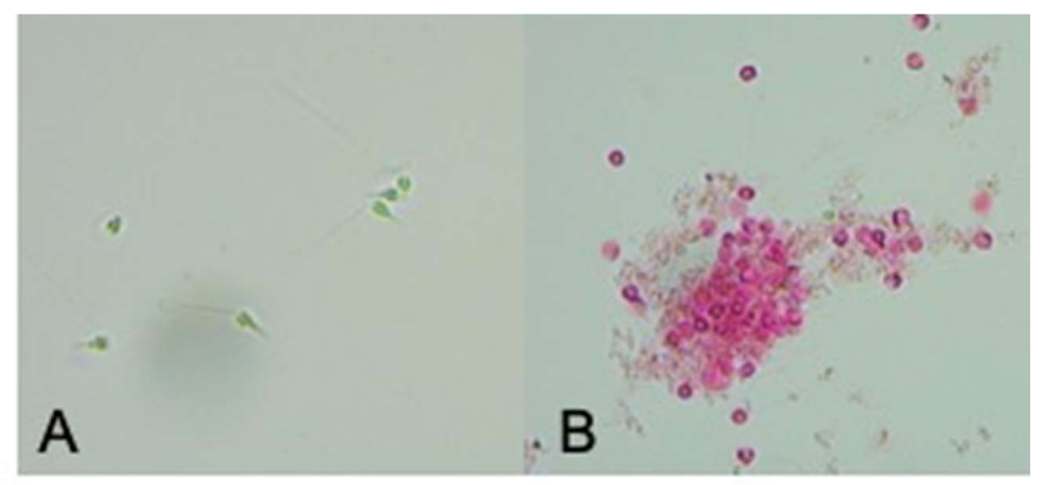

Figure 1. Vitality evaluation with Eosin Test on Mytilus galloprovincialis sperms. A) Untreated sample. B) After exposure to $27 \mathrm{GHz}$ for 30 minutes. 


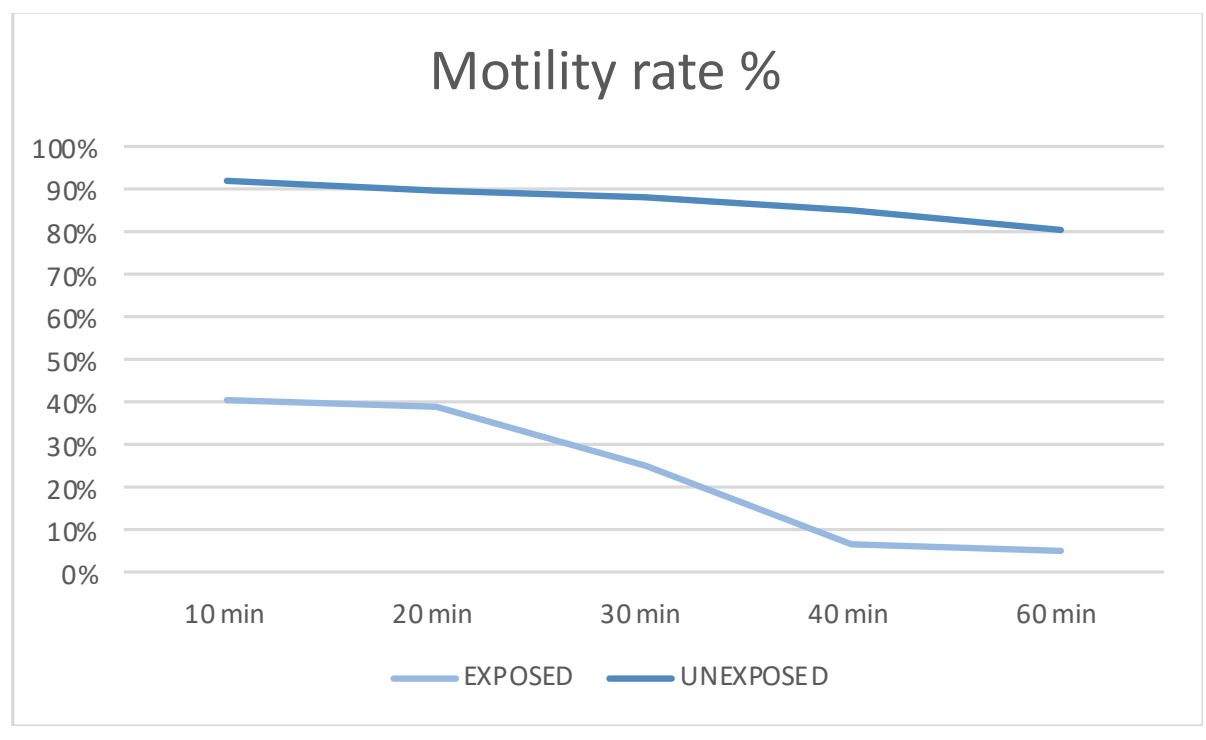

Figure 3. Temporal trends of the time-exposure decrease of sperms motility to $27 \mathrm{GHz}$ frequency. The data represent the mean of observation performed in tenfold by same observer to avoid subjective differences in motility evaluation.

\subsection{Oxidative stress analyses}

Oxidative stress has been evaluated with the MiOXSYS System. It is based on the measurement of the Oxidation Reduction Potential (sORP), an overall index of the electron passage to which a biological component is subjected. sORP is an integrated measure of all oxidants and reducing agents, which evaluates the oxidative damage that spermatozoa are undergoing due to free radicals. The analysis showed in the control groups a current passage of $2.33 \mathrm{mV} / 10^{6} \mathrm{sperm} / \mathrm{ml}$ and in exposed groups of $3.18 \mathrm{mV} / 10^{6} \mathrm{sperm} / \mathrm{ml}$. Therefore, is evident how the exposure to $27 \mathrm{GHz}$ of spermatozoa, increase the oxidant amount, compared to the control groups. This suggest that the antioxidant enzyme system of sperm cells was not able to counteract excessive presence of ROS, which, as shown by Liu et al., 2015 [8], increases following exposure of the spermatozoa to electromagnetic fields.

\section{Discussion}

Fifth generation of global mobile communications will bring also a new era to maritime connectivity for real-time data transmissions. Coastal environments are very biodiverse, but human activities, pollution and development along coastlines can stress these environments limiting the rates of growth and reproduction of coastal fauna. However, while much is present in the literature on the effect on reproduction of electromagnetic fields in terrestrial animals model [8-13], little is known about the effect in aquatic animals, except for a few data concerning zebrafish $[15,16]$.

Recent evidences demonstrate that electromagnetic fields negatively affect sperm quality, sperm count, morphology and motility. In male rats 2 days old, exposed to EMF 1800 and $900 \mathrm{MHz}$ for 2 hours continuously per day for 90 days. The percentage of epididymal sperm motility was significantly higher in the $1800 \mathrm{MHz}$ group $(\mathrm{p}<0.05)$. The morphologically normal spermatozoa rates were higher and the tail abnormality and total percentage abnormalities were lower in the $900 \mathrm{MHz}$ group $(\mathrm{p}<0.05)$ [9]. In another study conducted always on rats exposed to $900 \mathrm{MHz}$ for 8 weeks, Authors noticed statistically significant decrease of epididymal sperm counts in the exposed group $(\mathrm{p}<0.001)$, a significant decrease of sperm motility and a significant $(p<0.001)$ increase in the frequency percentage of dead spermatozoa [10]. Guo et al., 2019 [11], exposing rats for 1 month to $220 \mathrm{MHz}$ demonstrated a decreased sperm count and survival rate of sperm $(p<0.05)$, increased sperm abnormalities. Rats, exposed to $900 \mathrm{MHz}$ for 50 days, showed significant 
increase of the percentage of apoptotic sperm cells by $91.42 \%$ and a significant increase of the ROS concentration by $46.21 \%$ [8]. No differences in body weight and development among the groups were found in mice of both sexes in rats exposed to $2.45 \mathrm{GHz}$ for 2 $\mathrm{h} /$ day, 5 days/week for 5 consecutive weeks, starting the day after birth [14]. On zebrafish exposed to $3.5 \mathrm{GHz}$ RFR, specific absorption rate $(\mathrm{SAR}) \approx 8.27 \mathrm{~W} / \mathrm{Kg}$ from $6 \mathrm{~h}$ post fertilization (hpf) to $48 \mathrm{hpf}$, have not been revealed significant impacts on mortality, morphology or photomotor response, but only a modest inhibition of startle response suggesting some levels of sensorimotor disruptions [15]. In zebrafish's fertilized eggs exposed to 27 $\mathrm{GHz}$ frequency, $9.7 \mathrm{~mW} / \mathrm{cm}^{2}$ incident power density, $23 \mathrm{dbm}$ for $96 \mathrm{hpf}$ no significant impacts on mortality nor on morphology were highlighted because the exposed larvae showed a normal detachment of the tail, presence of heart-beat and well organised somites [16]. In our study we showed a notable decrease in the vitality and motility of $M$. galloprovincialis spermatozoa already after only 10 minutes of exposure to $27 \mathrm{GHz}$ and an increase in sORP values. Oxidation Reduction Potential was measured with the MiOXSYS System, which allows to way to directly assess the redox balance between ROS and antioxidants. A higher ORP corresponds to higher levels of oxidative activity [17], suggesting that the antioxidant enzyme system of the spermatozoa was unable to counteract the excessive presence of ROS; the excessive presence of ROS can also cause an increase in the concentration of proteins involved in cellular stress [18].

\section{Conclusions}

Our study indicates that electromagnetic fields a $27 \mathrm{GHz}$ can affect the sperm quality in Mytilus galloprovincialis. The significative decrease observed in sperm motility after only 10 minutes of exposure represents a crucial factor need to be considered because it can threat reproductivity of some species. This study provides useful data on potential ecological impact of the EMFs on aquatic animals, that currently are poor investigated. Direct and indirect stressors could affect the on coastal and marine biodiversity. Future research would benefit from specific investigation on the impact of $5 \mathrm{G}$ to better monitoring effects on animal organisms.

Author Contributions: Conceptualization, L.D.D. and M.V.B.; methodology, R.P., E.M.S, M.C., S.C.P. and G.S..; investigation, C.S. and S.I.; data curation, S.I. and C.S.; writing-original draft preparation, L.D.D. and M.V.B.; writing-review and editing, R.P., E.M.S. and M.V.B.; visualization, A.S.; supervision, L.D.D., R.P. and M.V.B..; funding acquisition, L.D.D. and M.V.B. All authors have read and agreed to the published version of the manuscript.

Funding: This research has been funded by "Programma ricerca di Ateneo UNICT 2020-22 linea 2".

Conflicts of Interest: The authors declare no conflict of interest.

\section{References}

1. Attaran, $\mathrm{M}$. The impact of $5 \mathrm{G}$ on the evolution of intelligent automation and industry digitization. J. Ambient. Intell. Human Comput. 2021. https://doi.org/10.1007/s12652-020-02521-x.

2. Foster, K.R.; Vijayalaxmi. Needed: More Reliable Bioeffects Studies at “High Band” 5G Frequencies. Front. Comms. Net. 2021, 2,721925 .

3. Hardell, L.; Carlberg, M. Health risks from radiofrequency radiation, including 5G, should be assessed by experts with no conflicts of interest. Oncol Lett. 2020, 20(4), 15.

4. Simkó, M.; Mattsson, M.O. 5G Wireless Communication and Health Effects-A Pragmatic Review Based on Available Studies Regarding 6 to $100 \mathrm{GHz}$. Int. J. Environ. Res. Public Health. 2019, 16(18), 3406.

5. Mattsson, M.O.; Simkó, M.; Foster, K.R. 5G New Radio Requires the Best Possible Risk Assessment Studies: Perspective and Recommended Guidelines. Front. Comms. Net. 2021, 2, 724772.

6. International Commission on Non-Ionizing Radiation Protection (ICNIRP), “Guidelines for limiting exposure to time-varying electric, magnetic and electromagnetic fields (up to $300 \mathrm{GHz}$ )". Health Phys. 1998, 74(4), 494-522.

7. Dreujou, E.; Desroy, N.; Carrière, J.; Tréau de Coeli, L.; McKindsey, C.W.; Archambault, P. Determining the Ecological Status of Benthic Coastal Communities: A Case in an Anthropized Sub-Arctic Area. Front. Mar. Sci. 2021, 8, 637546.

8. Liu, Q.; Si, T.; Xu, X.; Liang F.; Wang, L.; Pan S. Electromagnetic radiation at $900 \mathrm{MHz}$ induces sperm apoptosis through bcl2, bax and caspase-3 signaling pathways in rats. Reprod Health. 2015, 12, 65. 
9. Ozlem Nisbet, H.; Nisbet, C.; Akar, A.; Cevik, M.; Karayigit, M.O. Effects of exposure to electromagnetic field (1.8/0.9 GHz) on testicular function and structure in growing rats. Res Vet Sci. 2012, 93, 1001-5.

10. Bin-Meferij, M.M.; El-Kott, O.F. The neuroprotective effects of Moringa oleifera against mobile phone electromagnetic radiation-induced infertility in rats. Int J Clin Exp. Med. 2015, 8, 12487-97.

11. Bilgici, B.; Gun, S.; Avci, B.; Akar, A.; Engiz, B. What is adverse effect of wireless local area network, using $2.45 \mathrm{GHz}$, on the reproductive system? Int J Radiat Biol. 2018, 94, 1054-1061.

12. Saygin, M.; Asci, H.; Ozmen, O.; Cankara F.N.; Dincoglu D.; Ilhan I. Impact of 2.45 GHz microwave radiation on the testicular inflammatory pathway biomarkers in young rats: The role of gallic acid. Environ Toxicol. 2016, 31, 1771-1784.

13. Ozguner, M.; Koyu, A.; Cesur, G.; Ural, M.; Ozguner, F.; Gokcimen, A.; Delibas, N. Biological and morphological effects on the reproductive organ of rats after exposure to electromagnetic field. Saudi Medical Journal. 2005, 26, 405-410.

14. Sambucci, M.; Laudisi, F.; Nasta, F.; Pinto, R.; Lodato, R.; Lopresto, V.; Altavista, P.; Marino, C.; Pioli, C. Early life exposure to $2.45 \mathrm{GHz}$ WiFi-like signals: effects on development and maturation of the immune system. Prog Biophys Mol Biol. 2011, 107, 393-8.

15. Dasgupta, S.; Wang, G.; Simonich, M.T.; Zhang, T.; Truong, L.; Liu, H.; Tanguay, R.L. Impacts of high dose 3.5 GHz cellphone radiofrequency on zebrafish embryonic development. PLoS One. 2020, 15(7), e0235869.

16. Pecoraro, R.; Pavone, S.C.; Scalisi, E.M.; Ignoto, S.; Sica, C.; Capparucci, F.; Iaria, C.; Salvaggio, A.; Sorbello, G.; Di Donato, L.; Brundo M.V. Multimarker approach to evaluate the exposure to electromagnetic fields at $27 \mathrm{GHz}$ (5G) on Danio rerio larvae. J. Mar. Sci. Eng. 2022, Pre-Print ID: 54070.

17. Kohen, R.; Nyska, A. Oxidation of biological systems: oxidative stress phenomena, antioxidants, redox reactions, and methods for their quantification. Toxicol. Pathol. 2002, 30, 620-65.

18. Kang, S.J.; Kim, B.M.; Lee, Y J.; Chung, H.W. Titanium dioxide nanoparticles trigger p53-mediated damage response in peripheral blood lymphocytes. Environ. Mol. Mutag. 2008, 49, 399-405. 